\title{
Kinetics of Thermal Decomposition of Titanium Hydride Powder Using in situ High-temperature X-ray Diffraction (HTXRD)
}

\author{
Hugo Ricardo Zschommler Sandim*, Bruno Vieira Morante, Paulo Atsushi Suzuki
}

\author{
Departamento de Engenharia de Materiais, Faculdade de Engenharia Química de Lorena, \\ C.P. 116, 12600-970 Lorena - SP, Brazil
}

Received: November 16, 2004; Revised: April 6, 2005

\begin{abstract}
The thermal decomposition of titanium hydride powder ( $\delta$-phase) to titanium ( $\alpha$-phase) was investigated by means of thermogravimetric analysis (TGA) and high-temperature X-ray diffraction (HTXRD) in high vacuum. The $\delta$-to- $\alpha$ phase transformation was followed in situ by HTXRD at temperatures varying from room temperature up to $1000{ }^{\circ} \mathrm{C}$. The transformation was also analyzed as a function of time at isothermal conditions from 450 to $650{ }^{\circ} \mathrm{C}$. The results of TGA show that the decomposition of the titanium hydride becomes significant at about $450{ }^{\circ} \mathrm{C}$. Above $500{ }^{\circ} \mathrm{C}$ the decomposition is completed in times shorter than 50 minutes. The apparent activation energy for hydrogen desorption was found to be $63 \pm 6 \mathrm{~kJ} . \mathrm{mol}^{-1}$.
\end{abstract}

Keywords: titanium hydride, thermal decomposition, desorption kinetics, X-ray diffraction

\section{Introduction}

Metal hydrides are intended for many applications including the development of technologies for storage, production, and purification of hydrogen for clean energy generation ${ }^{1}$. Several metal powders including titanium, zirconium, niobium, and tantalum are commonly processed by the well-known hydride-dehydride (HDH) technique ${ }^{2}$. In the $\mathrm{HDH}$ process, metallic pieces (e.g. machining turnings, sponge fines or selected scrap) are embrittled by gaseous hydrogen yielding the corresponding hydrides. These brittle hydrides are milled, sieved, and dehydrided in vacuum at high temperatures to obtain the corresponding metallic powders. As a common feature, particles of these powders display the characteristic angular morphology. HDH-Ti, in particular, is commonly employed in the manufacture of low-cost powder metallurgy parts.

There are many different allotropic forms for titanium hydrides ${ }^{3,4}$, such as $\alpha, \beta$, and $\delta$-phases shown in the Ti-H phase diagram ${ }^{5}$ (see Figure 1), depending on the hydrogen concentration, temperature, and applied pressure. The accurate determination of the hydrogen site position in the crystal structure of these phases by X-ray diffractometry (XRD) is difficult due to the small scattering factor of the hydrogen. Some structural models shown in the literature were obtained from neutron diffraction measurements. At lower temperatures, e.g. below $600 \mathrm{~K}, \delta$-phase is commonly found when Ti is exposed to pure hydrogen. The $\delta$-phase has a $\mathrm{CaF}_{2}$-type crystal structure. It could be possible that vacancies are created in the F-site when the $\mathrm{H}$ :Ti ratio is smaller than 2 . For smaller hydrogen concentrations, it has been observed a phase transition to $\alpha$-phase, which crystallizes in a Mg-type crystal structure. The $\beta$-phase, which appears at higher temperatures, has a W-type crystal structure.

An interesting review concerning the kinetics of absorption and desorption reactions in hydrogen storage materials is found elsewhere ${ }^{6}$. According to the authors, hydrogen desorption in hydride-containing materials can be described as a sequence of partial steps including thermal decomposition of the hydride at the hydride-metal interface, interstitial diffusion of hydrogen through metal phase, surface penetration of hydrogen atoms, recombination of chemisorbed hydrogen atoms and physisorption, and molecular hydrogen degassing.
The desorption rate limiting steps as well as their corresponding activation energies depend on applied pressure, temperature, and surface contamination (oxidation, for instance). In most cases, the rate-limiting step cannot be determined in a straightforward manner. Gao and Huang have proposed that hydrogen desorption is not controlled by diffusion nor by any phase transformation (interface process), but by a process involving hydrogen transport through the surface oxide film? ${ }^{7}$.

The release of hydrogen resulting from the thermal decomposition of metal hydrides can be investigated by several techniques including thermogravimetric analysis (TGA), thermal desorption spectroscopy (TDS), differential thermal analysis (DTA) ${ }^{7-9}$, and on-time $\mathrm{H}_{2}$-pressure evaluation in a closed system with constant volume. In all cases, the main difficulty, however, is following the concentration changes occurring at the end of the dehydriding process. The residual amount of the hydrogen in the structure cannot be determined even by conventional XRD, since hydrogen is almost transparent for X-rays.

The hydrogen desorption kinetics can be determined by controlled heating of $\mathrm{TiH}_{2}$ in the powdered form in vacuum. XRD is a simple and versatile technique, which allows the observation of the $\delta$-to- $\alpha$ phase transformation. On the other hand, the study of its kinetics has to take into account the following limitations: a) the integration time, in the range of seconds, which limits the measurements at the earlier stages of the transformation (depending on the temperature); b) the lower detection limit (about 3\% in volume); and c) the low values of the heating rate, that does not allow reach the nominal temperature in short times. The conventional heating devices usually display heating rates ranging from 5 to $100{ }^{\circ} \mathrm{C} \cdot \mathrm{min}^{-1}$.

High-temperature X-ray diffraction (HTXRD) has been used to evaluate the desorption kinetics of $\delta-\mathrm{TiH}_{2}$ in electrochemicallycharged titanium sheets in a large interval of temperatures (up to $\left.600{ }^{\circ} \mathrm{C}\right)^{8}$. Within this framework, this technique can be also used to investigate the desorption kinetics in powdered $\delta$ - $\mathrm{TiH}_{2}$ in the temperature range comprised in the interval $450-650{ }^{\circ} \mathrm{C}$. From the industrial viewpoint, temperatures below $450{ }^{\circ} \mathrm{C}$ would demand longer desorption times whereas heating at temperatures above $650{ }^{\circ} \mathrm{C}$ has the 
Hydrogen (wt. (\%))

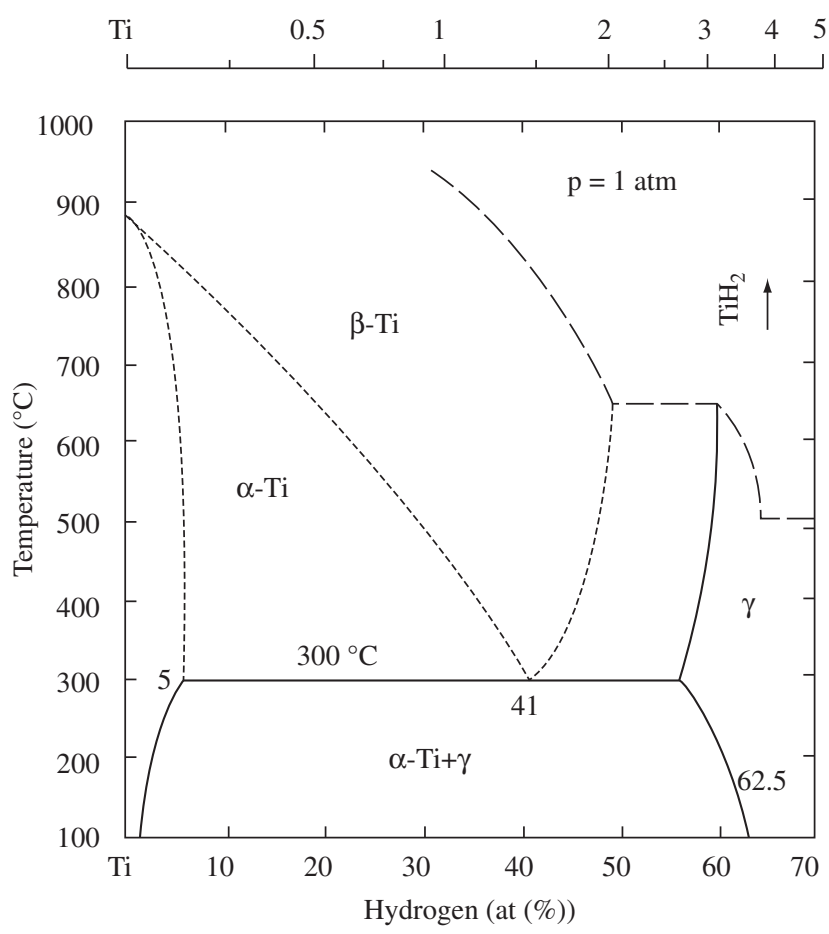

Figure 1. Ti-H phase diagram at $\mathrm{P}=1 \mathrm{~atm}$.

disadvantage of a higher energy expenditure. Thus, determining the hydrogen desorption kinetics in this particular system is a prerequisite for setting optimal conditions for processing low-cost titanium powders. In the present work we investigate the hydrogen desorption (in situ) in titanium hydride powder samples $\left(\delta-\mathrm{TiH}_{2}\right)$ using thermal gravimetric analysis (TGA) and conventional high temperature $\mathrm{X}$-ray diffraction (HTXRD).

\section{Experimental}

Titanium hydride powder was produced by controlled hydriding of Ti turnings at $550{ }^{\circ} \mathrm{C}$. The hydrided material was milled for 5 minutes at room temperature in a planetary ball mill under argon atmosphere. The X-ray diffractometry measurements were performed in a Seifert Isodebyeflex 1001 diffractometer with Ni-filtered $\mathrm{Cu}-\mathrm{K} \alpha$ radiation. XRD diffractograms of $\delta-\mathrm{TiH}_{2}$ and Ti were determined at room temperature in the $20^{\circ} \leq 2 \theta \leq 80^{\circ}$ angular interval with $0.05^{\circ}$ steps and 3 seconds counting time in order to determine the best $2-\theta$ interval for further HTXRD experiments. The structures of both $\mathrm{Ti}$ and $\delta-\mathrm{TiH}_{2}$ were refined using the Rietveld method (FullProf program ${ }^{10}$ ) in order to determine the corresponding lattice parameters. The hydrogen content in $\delta-\mathrm{TiH}_{2}$ powder was determined by conventional techniques.

The HTXRD experiments were carried out in a Bühler HDK2.4 high-temperature attachment and a Johanna Otto REP2000 temperature controller coupled to the diffractometer. A $1 \mathrm{~mm}$-thick layer of loose powder was placed on the top of a tantalum strip (about $0.5 \mathrm{~mm}$ thick), which was used as a primary heater by electrical current flow (Joule-effect heating). A Pt/PtRh10\% thermocouple was welded just below the tantalum strip to follow temperature upon heating. A cylindrical secondary heater was mounted around the strip in order to improve the temperature homogeneity. Grinding the $\delta-\mathrm{TiH}_{2}$ powder prior to place the powder on the tantalum strip minimized texture effects.
The $\delta$-to- $\alpha$ phase transformation was analyzed as function of time at isothermal conditions. The measurements were performed in the high-vacuum chamber and the temperature was set at 450, 500, 550, and $650{ }^{\circ} \mathrm{C}$ with a constant heating rate of $30^{\circ} \mathrm{C} \cdot \mathrm{min}^{-1}$. A turbomolecular pump provided vacuum close to $2 \cdot 0.10^{-5} \mathrm{mbar}$ at the beginning of the heating in all runs. The measurements were performed within the $34^{\circ} \leq 2 \theta \leq 36^{\circ}$ interval with a $0.05^{\circ}$ step and 1 second count. These conditions allow us to take a single measurement at every 2 minutes. This interval was chosen to follow the changes in the intensity of the most intense $\delta$-(111) and $\alpha-(010)$ reflections. The background was removed for the intensities analysis by subtracting a straight line below the peaks.

In order to estimate the proportion of $\delta$ and $\alpha$-phases (in wt. (\%)) during the transformation, the integrated intensities of the diffracted peaks were analyzed. According to Klug and Alexander ${ }^{11}$ the intensity of a phase as a function of its concentration in binary mixtures obeys a linear relationship when the X-ray absorbing power of the components is the same. In the present investigation, the absorbing power of both $\delta$ and $\alpha$-phases is almost the same, since hydrogen is quite transparent to X-rays. Therefore, it is reasonable to assume that the measured integrated intensity $(I)$ obeys a linear relation with the proportion of each phase. Since the $\delta$-(111) and $\alpha-(010)$ reflections are overlapped, a simple model was proposed for the analysis taking into account the concurrent contribution of both peaks. It is reasonable to assume that the measured integrated intensity $(I)$ obeys a linear relation with the proportion of each phase. Thus, it could be written as follows:

$$
\alpha-\text { phase }(\text { wt. }(\%))=\frac{\left(I-I_{\delta}\right) \cdot 100}{I_{\alpha}-I_{\delta}}
$$

where $I$ is the measured integrated intensity, $I_{\delta}$ is the integrated intensity for $100 \%$ of $\delta$-phase and, $I_{\alpha}$ is the integrated intensity for $100 \%$ of $\alpha$-phase. $I_{\delta}$ gives the integrated intensity calculated from the measurement of the titanium hydride before heating and $I_{\alpha}$ is the integrated intensity of the titanium calculated after the complete dehydriding process.

The morphology and the mean particle size of the powder used in this investigation were determined by analyzing a set of images obtained in a scanning electron microscope in the secondary electrons mode. The thermogravimetric analysis was performed in a Netzsch model STA 409C3 equipment. Measurements were performed under flowing high-purity argon with a heating rate varying from 5 to $20{ }^{\circ} \mathrm{C} \cdot \mathrm{min}^{-1}$.

\section{Results and Discussion}

The titanium hydride powder showed particles with the typical angular morphology because of the brittle character of fracture during milling and a mean particle size of about $35 \mu \mathrm{m}$. The X-ray diffractogram obtained for $\mathrm{TiH}_{1.924}$ powder used in the present investigation is shown in Figure 2a. All the reflections could be indexed as belonging to the $\delta$-phase. The refinement of the structure shows the agreement with the $\mathrm{CaF}_{2}$-type structure. The lattice parameter was found to be $a=4.456(2) \AA$. This value is close to the reported value $a=4.431 \AA$ for $\mathrm{TiH}_{185}{ }^{12}$. Figure $2 \mathrm{~b}$ shows the $\mathrm{X}$-ray diffraction profile obtained from the powder after the dehydriding process. All peaks belong to the $\alpha$-phase. The refined lattice parameters were: $a=2.950(2) \AA$ and $c=4.689(2) \AA$. These values are very close to the reported ones: $a=$ $2.9508 \AA$ and $c=4.6855 \AA^{12}$.

The results of TGA of the titanium hydride powder are shown in Figure 3. The total initial amount of hydrogen in the hydride powder was found to be close to $2.91 \pm 0.16 \mathrm{wt}$. $(\%)$. It corresponds to a composition of about 60 at $(\% \mathrm{H})$. The weight loss becomes noticeable between 450 and $500{ }^{\circ} \mathrm{C}$ and it can be attributed mainly to the 


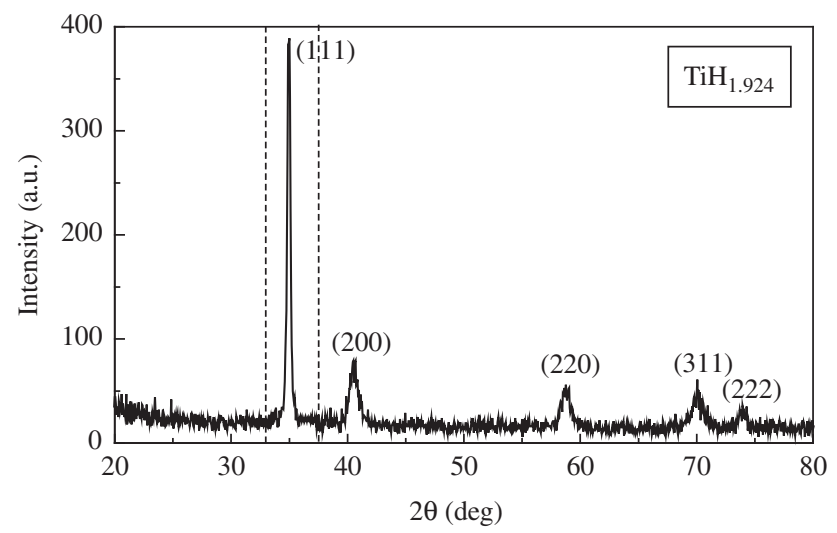

(a)

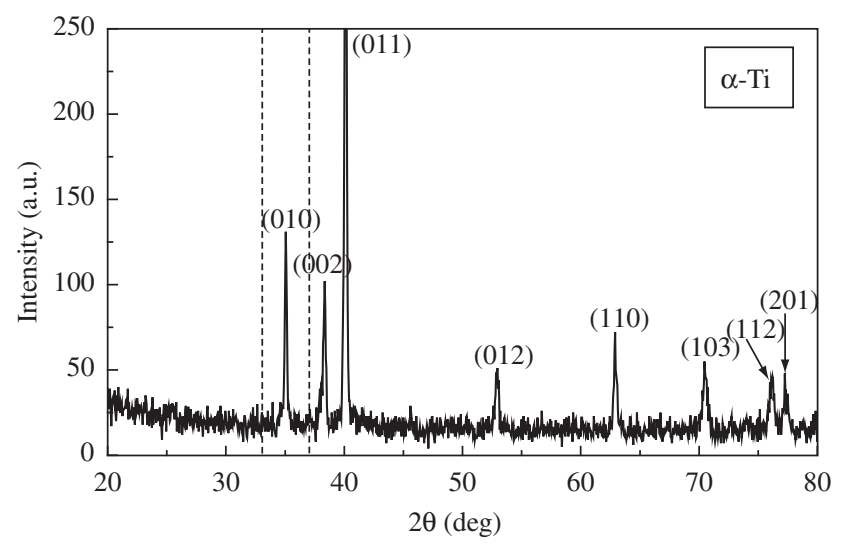

(b)

Figure 2. X-ray diffractograms corresponding to: a) the $\mathrm{TiH}_{1.924}$ powder; b) $\alpha$-phase after dehydriding process. The window for HTXRD measurements is marked with dashed lines.

hydrogen desorption. The onset temperature for hydrogen release was found to depend on the heating rate.

Results show that the lower the heating rates the lower is the onset temperature. The final amount of hydrogen in the transformed powder was found to be also dependent on the applied heating rate, as expected. Lower heating rates enable larger residence times favoring a more pronounced degassing.

The evolution of the $\delta$-to- $\alpha$ phase transformation as function of the temperature is shown in Figure 4. At temperatures below $500{ }^{\circ} \mathrm{C}$, only peaks belonging to the $\delta$-phase are observed. At higher temperatures, above $550{ }^{\circ} \mathrm{C}$, the transformation to $\alpha$-phase becomes noticeable. The small displacement of the $\alpha$-phase peaks to lower angles as a function of the temperature can be attributed to thermal expansion effects. It is worthwhile mentioning that the diffractograms shown in this paper depict a non-equilibrium condition. This is because the dehydriding process already occurs while sample is heated up before temperature reaches the programmed value. Therefore, part of hydrogen is released before nominal temperature is reached. This effect is more pronounced when measurements are made at higher temperatures and cannot be neglected. However, the selected temperature range used in this investigation allowed us to follow the dehydriding process close to the temperatures normally used in industrial processes (i.e. 500 to $600{ }^{\circ} \mathrm{C}$ ). At lower temperatures, kinetics is not favored whereas at higher ones hydrogen desorption occurs in a very fast manner making the use of conventional XRD a hard task due to its low integration time.

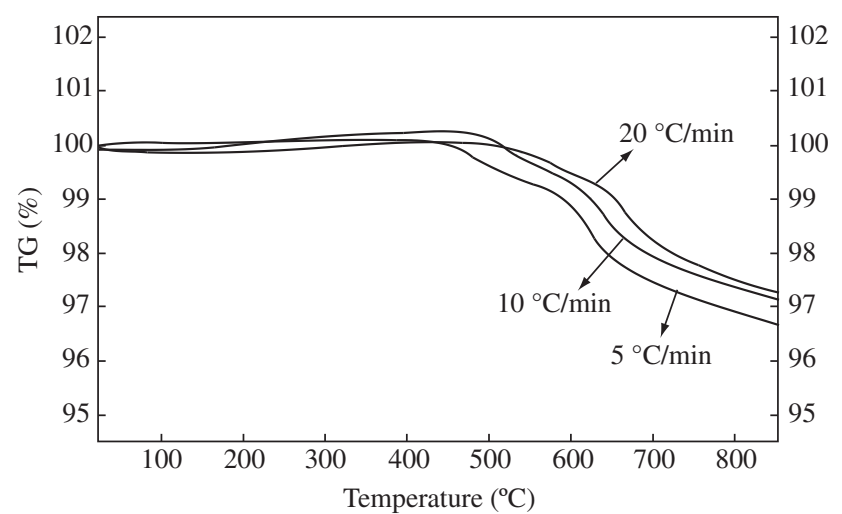

Figure 3. TGA curves for $\delta-\mathrm{TiH}_{2}$ powder for heating rates varying from 5 to $20{ }^{\circ} \mathrm{C} \cdot \mathrm{min}^{-1}$.

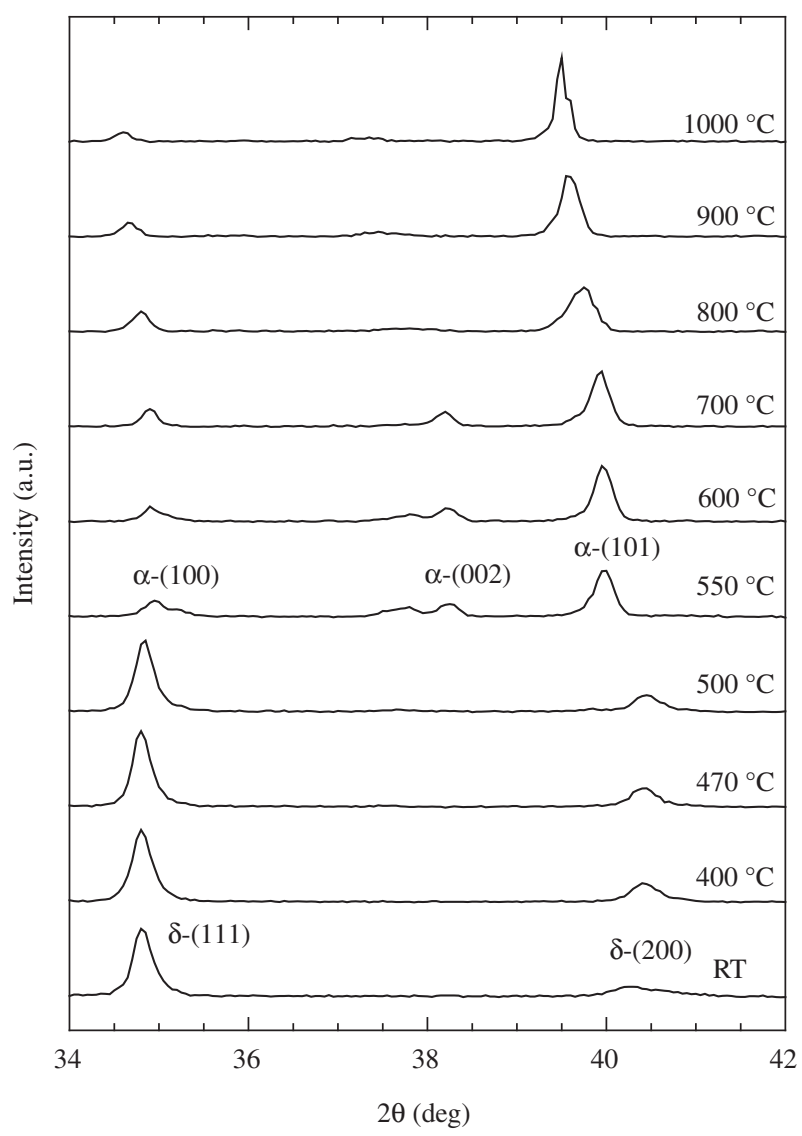

Figure 4. X-ray diffractograms showing the evolution of $\delta$-to- $\alpha$ phase transformation as a function of temperature.

In the intermediate dehydriding condition (close to $500{ }^{\circ} \mathrm{C}$ ), it is expected the appearance of peaks belonging to both $\delta$ and $\alpha$ phases. In this case, the most intense $\delta$-(111) peak will be overlapped with the $\alpha-(100)$ peak and the most intense $\alpha-(101)$ peak will be overlapped with the $\delta$-(200) peak. This overlap makes difficult the estimation of the percentage of each phase. At higher temperatures, desorption kinetics is too fast and cannot be followed properly by conventional HTXRD. Most accurate hydrogen desorption kinetics data were recently obtained by means of time-resolved XRD using synchrotron radiation, as reported by Huot for a similar system $\left(\mathrm{MgH}_{\mathrm{x}}\right)^{13}$. 
Figure 5 shows the proportion of the $\alpha$-phase calculated from Equation 1 as function of time for different temperatures. A sigmoidal function fits the experimental data in good agreement. The accuracy of the data is higher for lower temperatures (below $550{ }^{\circ} \mathrm{C}$ ). For a better comparison of the data, the time needed for $50 \%$ transformation $\left(\mathrm{t}_{05}\right)$ from $\delta$ to $\alpha$-phase is displayed in the Figure 6 . The apparent desorption activation energy was calculated using the Arrhenius-type equation:

$$
\ln \left(t_{0.5}\right)=-\left(E / k_{\mathrm{B}} T\right)+A
$$

where $t_{0.5}$ is the time for $50 \%$ transformation, $E$ is the activation energy, $k_{\mathrm{B}}$ is the Boltzmann constant, $T$ is the absolute temperature and $A$ is a constant. The activation energy was calculated from the slope of the $\ln \left(t_{0.5}\right) v s .(1 / T)$ plot, resulting in a value close to $63 \pm 6 \mathrm{~kJ} . \mathrm{mol}^{-1}$. This value is close to that one reported for the heat of decomposition of titanium hydride $\left(\sim 67 \mathrm{~kJ} \mathrm{~mol}^{-1}\right)^{14}$ and slightly higher than the activation energy for diffusion of hydrogen in $\alpha$-titanium $\left(42-54 \mathrm{~kJ}^{-\mathrm{mol}^{-1}}\right)^{7}$. It is worth noting that these values depend on the technique employed for its determination.

Gao and Huang ${ }^{7}$ have also reported a value of $67 \mathrm{~kJ}^{\mathrm{m}} \mathrm{mol}^{-1}$ for the apparent activation energy for hydrogen desorption in titanium. They have used thin sheets of titanium instead of titanium powder and suggest that the desorption rate is controlled by hydrogen transport

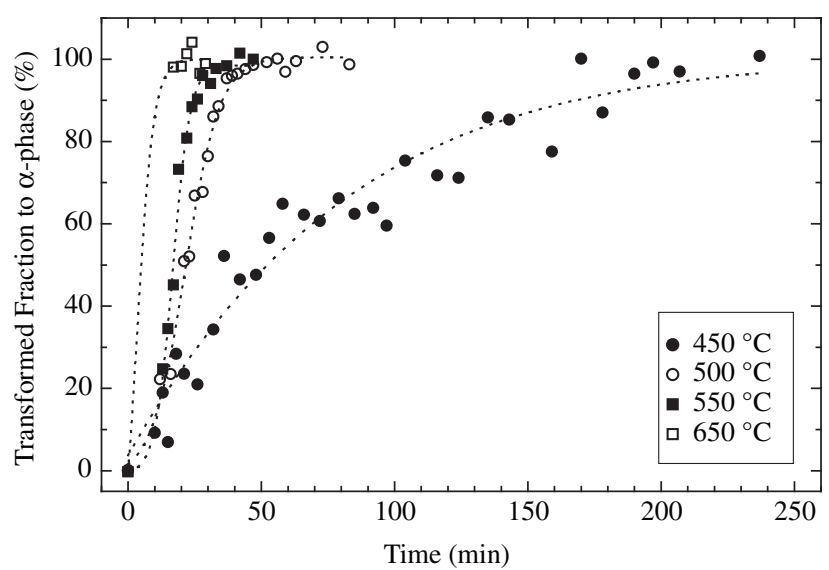

Figure 5. $\delta$-to- $\alpha$ transformation kinetics for temperatures between 450 and $650{ }^{\circ} \mathrm{C}$.

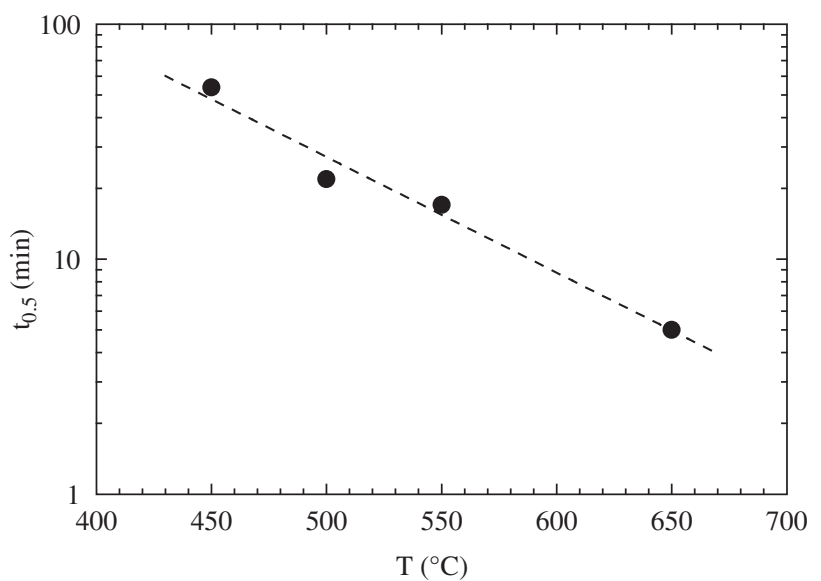

Figure 6. Time for $50 \%$ transformation $\left(\mathrm{t}_{0.5}\right)$ as a function of the dehydriding temperature. through the surface oxide film based on the visual inspection of the heated sheets (formation of an oxide layer).

Takasaki et al. ${ }^{8}$ have investigated the dissociation behavior of titanium hydride in bulk specimens prepared by electrochemical charging of $\alpha$-titanium sheets. They have reported values of the apparent activation energy for $\delta$-hydride dissociation and hydrogen evolution varying from $106 \mathrm{~kJ} . \mathrm{mol}^{-1}$ using DTA to a lower value, close to $49 \mathrm{~kJ} . \mathrm{mol}^{-1}$, estimated by means of thermal desorption spectroscopy (TDS). The last value has been calculated using the Kissinger method for TDS analyses; however, it is worth mentioning the number of experimental points was limited (three at all).

In addition to the non-equilibrium conditions at the beginning of the dehydriding experiments, Martin et al. ${ }^{6}$ also points out that possible deviations of non-isothermal conditions are due to the heat released during phase transformations and contamination effects. The latter might strongly affect the kinetics of hydrogen desorption, even when interstitials are present in very low contents ${ }^{15}$. This aspect is relevant since titanium is a reactive metal; however, oil-free dynamic vacuum conditions were established in our experiments minimizing carbon pick-up at least. Nevertheless, poisoning due to interstitials like oxygen and nitrogen cannot be fully prevented even using a turbomolecular pump during the HTXRD measurements.

The results presented in this paper show that HTXRD and TGA can be used to follow the thermal decomposition of $\delta$ - $\mathrm{TiH}_{2}$ into $\alpha$-Ti during heating in vacuum (dynamic conditions). The apparent activation energy for hydrogen desorption in $\delta-\mathrm{TiH}_{2}$ has been evaluated by HTXRD measurements $\left(63 \pm 6 \mathrm{~kJ} \cdot \mathrm{mol}^{-1}\right)$. This value is similar to those reported by many authors using other characterization techniques including TDS, DTA, and TGA. However, it is not possible to identify which mechanism is the rate-controlling step for hydrogen desorption in an unambiguous manner by a simple analysis of the apparent activation energy. The hypothesis of a surface-controlled mechanism for hydrogen desorption seems to be the most plausible. Further experimental investigation is still necessary to clarify this point, in particular the structure and composition of the surface layer formed during progressive poisoning of titanium.

\section{Conclusions}

The evolution of the $\delta$-to- $\alpha$ phase transformation has been investigated by high temperature X-ray diffraction (HTXRD). The onset temperature for hydrogen release from $\delta$-hydride was found to depend on the heating rate in TGA. The decomposition of the $\delta$-phase can be followed by HTXRD at temperatures above $450{ }^{\circ} \mathrm{C}$. Above $500{ }^{\circ} \mathrm{C}$ the kinetics of hydrogen desorption is faster and is completed in times shorter than 50 minutes. The apparent activation energy for hydrogen desorption in $\delta-\mathrm{TiH}_{2}$ was estimated to be $63 \pm 6 \mathrm{~kJ} \cdot \mathrm{mol}^{-1}$.

\section{Acknowledgments}

Authors are thankful to FAPESP for the financial support and to Dr. J. Otubo (CTA, Brazil) for his kind assistance on TGA experiments. CNPq (Brazil) supports H. R. Z. Sandim.

\section{References}

1. Sandrock GA. A panoramic overview of hydrogen storage alloys from a gas reaction point of view. Journal of Alloys and Compounds. 1999; 293-295:877-888.

2. German RM. Powder Metallurgy Science. Princeton: MPFI; 1984.

3. Numakura H, Koiwa M. Hydride precipitation in titanium. Acta Metallurgica. 1984; 32(10):1799-1807.

4. Woo OT, Weatherley GC, Coleman CE, Gilbert RW. The Precipitation of gamma-deuterides (hydrides) in titanium. Acta Metallurgica. 1985; 33(10):1897-1906. 
5. Massalski TB, Subramanian PR, Okamoto H, editors. Binary Alloy Phase Diagrams. $2^{\text {nd }}$ edition. Ohio: ASM International, Metals Park; 1986.

6. Martin M, Gommel C, Bokhart C, Fromm E. Absorption and desorption kinetics of hydrogen storage alloys. Journal of Alloys and Compounds. 1996; 238(1-2):193-201.

7. Gao S-J, Huang L-J. Hydrogen absorption and desorption by Ti, Ti-5Cr and Ti-5Ni alloys. Journal of Alloys and Compounds. 1999; 293-295:412-416.

8. Takasaki A, Furuya Y, Ojima K, Taneda Y. Hydride dissociation and hydrogen evolution behavior of electrochemically charged pure titanium. Journal of Alloys and Compounds. 1995; 224(2):269-273.

9. Chen Y, Williams JS. Formation of metal hydrides by mechanical alloying. Journal of Alloys and Compounds. 1995; 217(2):181-184.

10. Rodriguez-Carvajal J. Reference Guide for the Computer Program FullProf. Saclay-France: Laboratoire Leon Brillouin CEA-CNRS; 1996.
11. Klug HP, Alexander LE. X-Ray Diffraction Procedures. $2^{\text {nd }}$ edition. New York: Wiley-Interscience; 1974. p.534-536

12. Villars P, Calvert LD, editors. Pearson's Handbook of crystallographic data for intermetallic phases. $2^{\text {nd }}$ edition. Ohio: ASM International, Metals Park; 1991.

13. Huot J, Pelletier JF, Lurio LB, Sutton M, Schulz R. Investigation of dehydrogenation mechanism of $\mathrm{MgH}_{2}-\mathrm{Nb}$ nanocomposites. Journal of Alloys and Compounds. 2003; 348(1-2):319-324.

14. Fromm E, Hörz G. Hydrogen, nitrogen, oxygen and carbon in metals. International Metallurgy Review. 1980; 5-6:269-311.

15. Filimonov IN, Yuschenko VV, Smirnov AV, Nesterenko SN, Dobryakova IV, Ivanova II, Lubnin EN, Galperin L, Jensen RH. Deactivation of titanium during temperature-induced hydrogen absorption-desorption cycling: I. Effects of water, oxygen and nitrogen traces. Journal of Alloys and Compounds. 2005; 390(1-2):144-154. 